\title{
The Entrance Region of Circular Pipes Revisited
}

\author{
Frederick J. Young \\ Ph.D. Retired Professor at CMU, 800 Minard Run Road, Bradford, PA, USA \\ Email: youngfjphd@gmail.com
}

Received 22 April 2016; accepted 15 July 2016; published 20 July 2016

Copyright (C) 2016 by author and OALib.

This work is licensed under the Creative Commons Attribution International License (CC BY). http://creativecommons.org/licenses/by/4.0/

(c) (i) Open Access

\begin{abstract}
The laminar flow in the entrance region of pipes having a circular cross section is investigated by the use of finite element solutions to the full Navier-Stokes equations in cylindrical coordinates. Because these solutions cover the complete three-dimensional geometry the usual development length is no longer a very interesting parameter as explained herein. The velocities and pressures are determined throughout the pipe and presented in graphic form.
\end{abstract}

\section{Keywords}

Flow Development, Round Pipe, Incompressible Flow, Constant Temperature, Complete Finite Element Solution, Navier-Stokes Equations

\section{Subject Areas: Applied Physics}

\section{Introduction}

In the last century many investigators have attempted to analyze the flow in the entrance of pipes. There was a limited amount of experimental work due to the difficulties encountered in measurement before electronics were well developed. An early work by Schiller [1] treated the problem in an approximate method. In the early days of digital computing Bodoia and Osterle [2] tried to solve the Navier-Stokes equations subject to many assumptions to make possible a finite difference solution. Campbell, W. D. and Slattery, J. C. [3] modified Schiller's method. Later Gerrard and Taylor [4] investigated blood flow in a normal artery. A numerical solution to the partial differential equations averaged over each section of the tube was used and these equations were solved by the method of finite differences making an entrance-length effect correction by Hornbeck [5].

When his work was done the most advanced computers were very slow and it was almost impossible to have more than 1/4 megabyte of storage. If one wanted to work on Christmas or New Year day a megabyte might be available for one program. Hornbeck [5] filled several rooms with IBM cards for his multitudinous computer runs over a period of several years. It seems to this author the treatment of the radial components of velocity 
given in his equations may be inadequate for a correct solution. Durst [6] et al. found a maximum value in the fluid velocity along the center of a round pipe that occurred after the velocity had reached $99 \%$ of its fully developed value. Toppel [7] et al. included heat transfer in the simulation of hydrodynamic flows in pipe networks. There are numerous other papers on flow development and they all suffer the difficulties cited by Hinsen [8]. Thus it is the goal of this paper to solve the full incompressible Navier-Stokes equations numerically using better hardware and software. In many investigations the computer program is not given and the reader cannot know exactly what was computed, how it was done and may not be able to repeat or alter the calculations. For these reasons the FLEXPDE program available at www.pdesolutions.com is given here.

\section{Fluid Equations for a Circular Pipe}

Below are the equations Hornbeck [5] solved to determine the entrance length in circular pipes. The first is the z-component of the Navier-Stokes equations and the second a statement of continuity.

$$
\begin{gathered}
\rho(u \partial u / \partial z+v \partial u / \partial r)=-\partial p / \partial z+(\mu / r) \partial / \partial r(r \partial u / \partial r) \\
\partial u / \partial z+(\mu / r) \partial / \partial r(r v)=0
\end{gathered}
$$

where $z$ is the axial coordinate, $r$ is the radial coordinate and $u$ and $v$ are the velocity components in the $z$ and $r$ directions respectively. $p$ is the fluid pressure, $\rho$ the fluid density and $\mu$ the fluid viscosity. Choosing dimensionless variables defined as

$$
U=u / u_{m}, \quad V=\rho v a / \mu, \quad R=r / a, Z=z \mu /\left(a \rho u_{m}\right) \text { and } P=\left(p-p_{0}\right) /\left(\rho u_{m}^{2}\right)
$$

where $a$ is the pipe radius and $u_{m}$ is the mean axial velocity. Then the full Navier-Stokes equations solved here are given by

$$
\begin{gathered}
\nabla \cdot \nabla U-\partial P / \partial Z-U \partial U / \partial Z-V \partial U / \partial R=0 \\
\nabla \cdot \nabla V-\partial P / \partial R-U \partial V / \partial Z-V \partial V / \partial R=0 \\
\nabla \cdot \nabla P=\text { penalty } \times \nabla U
\end{gathered}
$$

Cylindrical coordinates are used and plots are presented where the radial coordinate, $R$ lies in the horizontal direction whilst the axial coordinate, $Z$ is vertical. The geometry is given in Figure 1 with the initial finite ele-

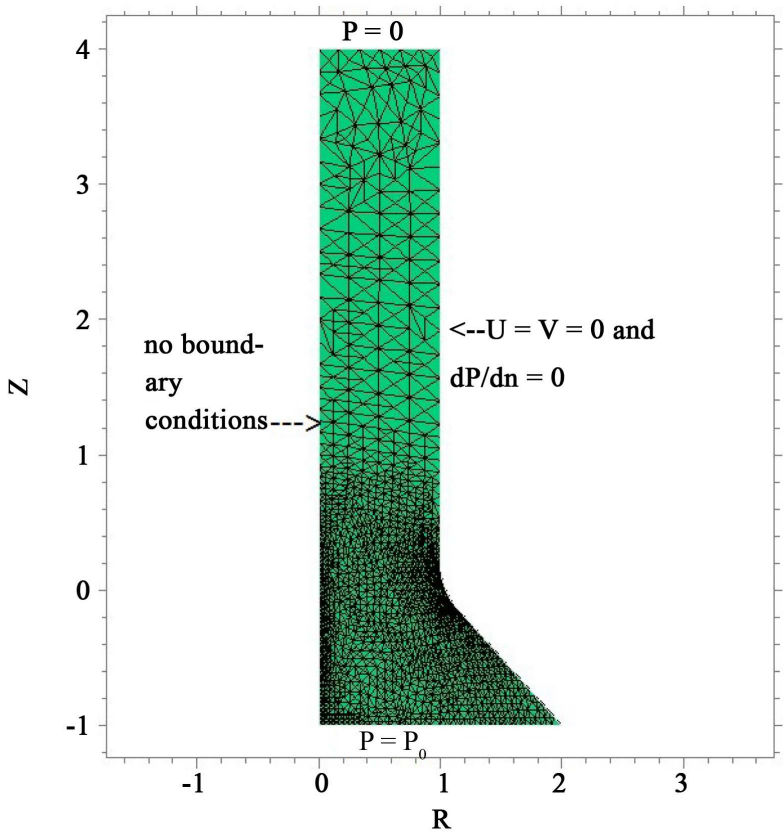

Figure 1. The pipe geometry, initial conditions and initial finite elements. 
ment mesh shown. The boundary conditions are also exhibited. When there are no conditions mentioned on aside of the figure the boundaryconditionsarenotspecifiedandaredeterminedbythegivenconditionsthatmay be on other boundaries. The center of the pipe is on the line $R=0$. In the straight part of the pipe the elements are uniformly distributed. In the flared part more elements are needed in the region where the slope of the pipe wall changes abruptly.

\section{The Computer Program to Solve the Navier-Stokes Equations in Cylindrical Coordinates}

TITLE "Flow Development Problem in Round Pipe"

\section{COORDINATES}

1. ycylinder ("r", "z")

\section{VARIABLES}

\section{U V P}

\section{SELECT}

3. cubic $=$ on

4. errlim $=0.0001$

\section{DEFINITIONS}

5. $\mathrm{P} 0=50$ penalty $=500$

6. $\mathrm{h}=4 \mathrm{w}=\operatorname{vector}(\mathrm{V}, \mathrm{U})$

7. u ex $=-2.73 *\left(\mathrm{r}^{\wedge} 2^{-1}\right)$

8. $\operatorname{div} \mathrm{U}=(\mathrm{dz}(\mathrm{u})+(1 / \mathrm{r}) * \mathrm{dr}(\mathrm{r} * \mathrm{v}))$

\section{EQUATIONS}

9. u: $\operatorname{div}(\operatorname{grad}(\mathrm{u}))-\mathrm{dz}(\mathrm{p})-\mathrm{u}^{*} \mathrm{dz}(\mathrm{u})-\mathrm{v}^{*} \operatorname{dr}(\mathrm{u})=0$

10. $\mathrm{v}: \operatorname{div}(\operatorname{grad}(\mathrm{v}))-\operatorname{dr}(\mathrm{p})-\mathrm{v} * \operatorname{dr}(\mathrm{v})-\mathrm{u}^{*} \mathrm{dz}(\mathrm{v})=0$

11. $\mathrm{p}: \operatorname{div}(\operatorname{grad}(\mathrm{p}))=$ penalty* $\operatorname{div} U$

\section{BOUNDARIES}

\section{Region 1}

12. $\operatorname{start}(0, \mathrm{~h}) \operatorname{nobc}(\mathrm{U}) \operatorname{nobc}(\mathrm{V}) \operatorname{nobc}(\mathrm{P})$ line to $(0,-1)$

13. value $(\mathrm{P})=\mathrm{P} 0$ line to $(2,-1)$

14. $\operatorname{value}(\mathrm{U})=0$ value $(\mathrm{V})=0$ natural $(\mathrm{P})=0$ line to $(1,0)$ fillet $(0.5)$ to $(1, \mathrm{~h})$

15. value $(\mathrm{P})=0 \operatorname{nobc}(\mathrm{U}) \operatorname{nobc}(\mathrm{V})$ line to close

\section{MONITORS}

16 contour(U) contour(V)

17. elevation(U) from $(-1,0)$ to $(-1,2)$ report(integral( $\left.\left({ }^{*} \mathrm{r}\right)\right)$

18. elevation(u-u_ex) from $(h, 0)$ to $(h, 1)$ elevation(u, u_ex) from $(0,0.98)$ to $(1,0.98)$

19 . vector(w) surface(U/globalmax(U)) viewpoint $(2.5,5,80)$ as "relative axial velocity"

\section{PLOTS}

20. contour (U) report(integral( $(\mathrm{U} * \mathrm{r}))$ contour $(\mathrm{V})$ contour $(\mathrm{P})$ vector(w) norm vector(w)

21. elevation (U) from $(0,-1)$ to $(2,-1)$ report(integral $\left.\left(U^{*} \mathrm{r}\right)\right)$

22. contour $(\mathrm{U})$ zoom $(0.8,0,1.3,0.5)$

23. elevation (U) from $(0,0.5)$ to $(0, \mathrm{~h}) \operatorname{report}(0.99 *$ globalmax $(U))$ elevation(U) from $(0,-1)$

24. to $(0,0.62 * h)$ report(integral( $\left.\left(U^{*}\right)\right)$ report $(0.99 * \operatorname{globalmax}(\mathrm{U}))$ elevation(u,u_ex) from

25. $(0, \mathrm{~h})$ to $(1, \mathrm{~h})$ elevation(u, u_ex) from $(0,0.62 * \mathrm{~h})$ to $(1,0.62 * \mathrm{~h})$ contour(divU) as "Continuity Check"

26. surface (U/globalmax(U)) viewpoint $(2.5,5,80)$ as "relative axial velocity"

27. surface (U) viewpoint $(2.5,5,80)$ as "axial velocity"

END

\section{Explanation of the Program}

Comments coming after the ! glyphs have been made in the program to show what each program line does. The program ignores anything on a line preceded by an!. In most cases there is an explanation of that command. In lines 5 and 6 the pressure at $Z=0$ is set arbitrarily and the pipe length is set to 4 dimensionless units. Here the pressure is $P=P_{0}$ and the other boundary conditions need not be specified. Line 7 is the equation of fully developed flow. Line 8 calculates the divergence of the flow to check the solution accuracy. 


\section{1) Setting up the partial differential equations}

Equations (3)-(5) are setup in lines 9, 10 and 11 where $\mathrm{d} Z(\zeta)=\partial \zeta / \partial Z$ and $\mathrm{d} R(\partial)=\partial \zeta / \partial R$. Here div and grad are defined for cylindrical coordinate systems.

\section{2) Setting the pipe geometry and establishing boundary conditions}

The center of the pipe lays on the $R=0$ axis and needs no boundary conditions as denoted by nobc (). The axis starts at $(0, h)$ and runs to $(0,-1)$ being $1+h$ units long. This is accomplished in line 12 above. The bottom or entrance of the pipe is defined in line 13 as going from the last mentioned point to $(2,-1)$. In line 14 the outside of the pipe is defined. It runs from the previously defined point to $(1,0)$ and to $(1, h)$ with a fillet of radius 0.5 where the funnel connects to the pipe. The rounding of the geometry near $(1,0)$ makes fewer finite elements necessary and eliminates undefined partial derivatives. Along that boundary the no slip condition dictates $U=0$ and obviously $V=0$. The normal partial derivative is given by $\partial P / \partial n=0$ along the funnel and the pipe inner wall. In line $15 P=0$ and no boundary conditions are needed for the fluid velocities as the boundary goes from $(1, h)$ to $(0, h)$, the point of beginning.

\section{3) Monitors and plots}

In lines 16 through 19 there are monitors that appear during the calculation to help determine if the program is yielding sensible results. Contours display the contours of $U$ and $V$ as a set of lines. Elevation gives a line plot of the variable over the range specified in the form () to () statement first given in line 17. In lines 21 and 22 report $(0.99 *$ globalmax $(U))$ exhibits the $99 \%$ of the largest velocity in the $Z$ direction. Here the last elevation plot of line 22 compares the solution for velocity obtained at $Z=1.62$ to the exact solution for fully developed circular pipe flow.

\section{4) Results}

In Figure 2 the contours of constant velocity in the $Z$ direction are shown. Clearly fully developed flow begins when $Z>1$. In Figure 3 the flow vectors are represented. The arrows indicating the direction of flow are all the same size and the velocities they represent are given by their color. They indicate full development when $Z>$ 1. As indicated in the upper left corner of Figure 3 it took 3 minutes and 22 seconds to do the finite element calculation described here. Figure 4 shows the contours of flow velocity in the radial direction and as would be expected there is much radial flow in the funnel region tapering to nearly zero as $Z \rightarrow 1$. The contour lines of pressure are shown in Figure 5 and are as expected. To check the validity of the solutions attained herein the divergence of the velocity is calculated in the pipe and funnel and exhibited in Figure 5 . It is close to zero in the whole geometry. The 99\% of the maximum speed in the $Z$ direction first occurs at $Z 0.62$ and Figure 6 exhibits a plot of a fully developed and the calculated profile of $U$ at $Z=0.62$. The area under the two curves differs by only 0.195 percent indicating the almost full development of the velocity profile in the pipe as evaluated at $Z=$ 0.62 . Figure 7 is the velocity, $U$ along the center line of the pipe. A peak in the velocity occurs before the flow is

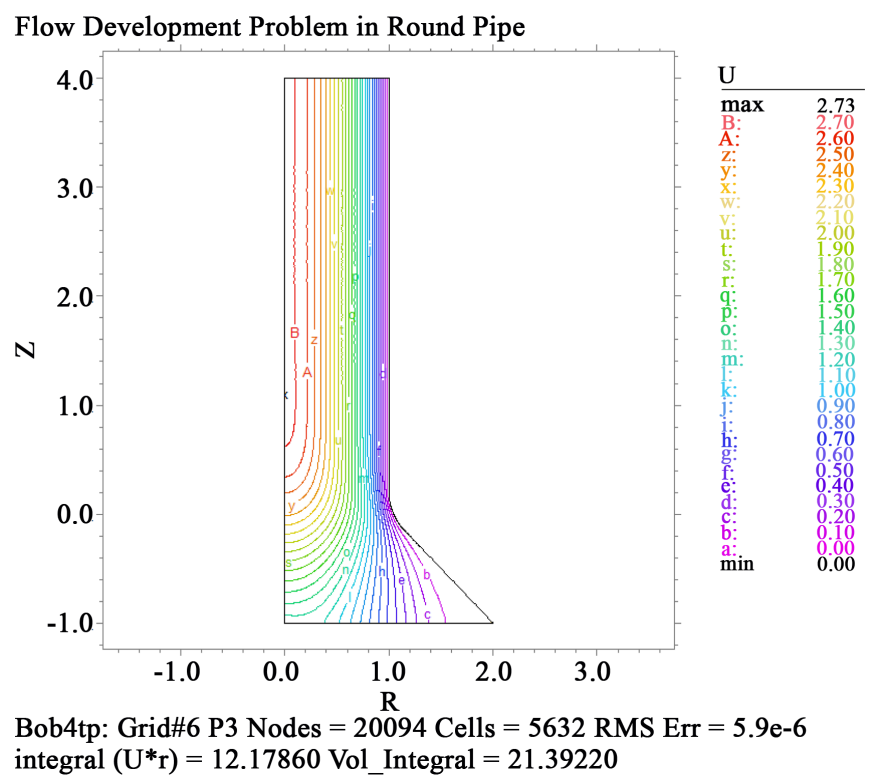

Figure 2. Pipe geometry with flow lines of the velocity. 


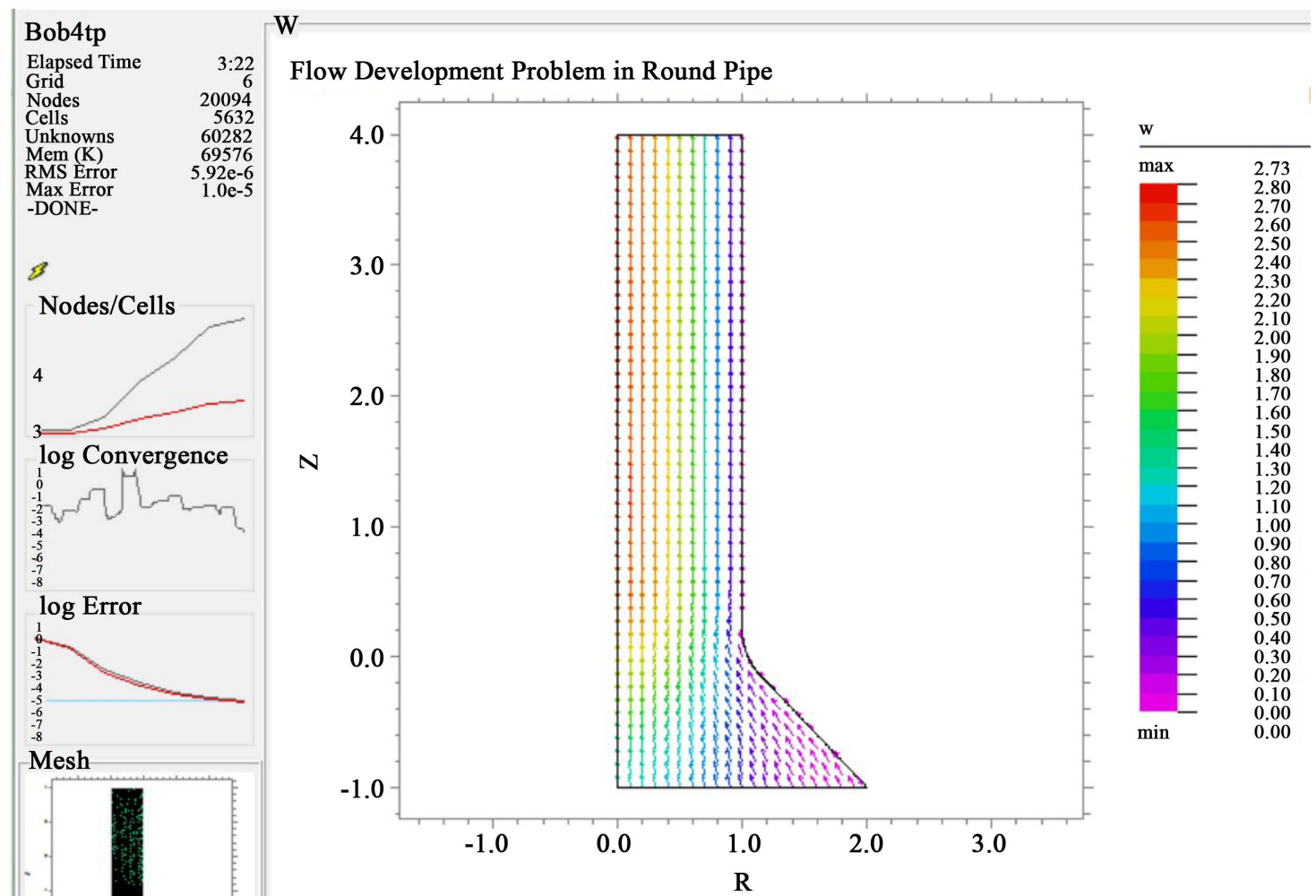

Bob4tp: Grid\#6 P3 Nodes $=20094$ Cells $=5632$ RMS Err $=5.9 \mathrm{e}-6$

Figure 3. Fluid flow vector.

Flow Development Problem in Round Pipe

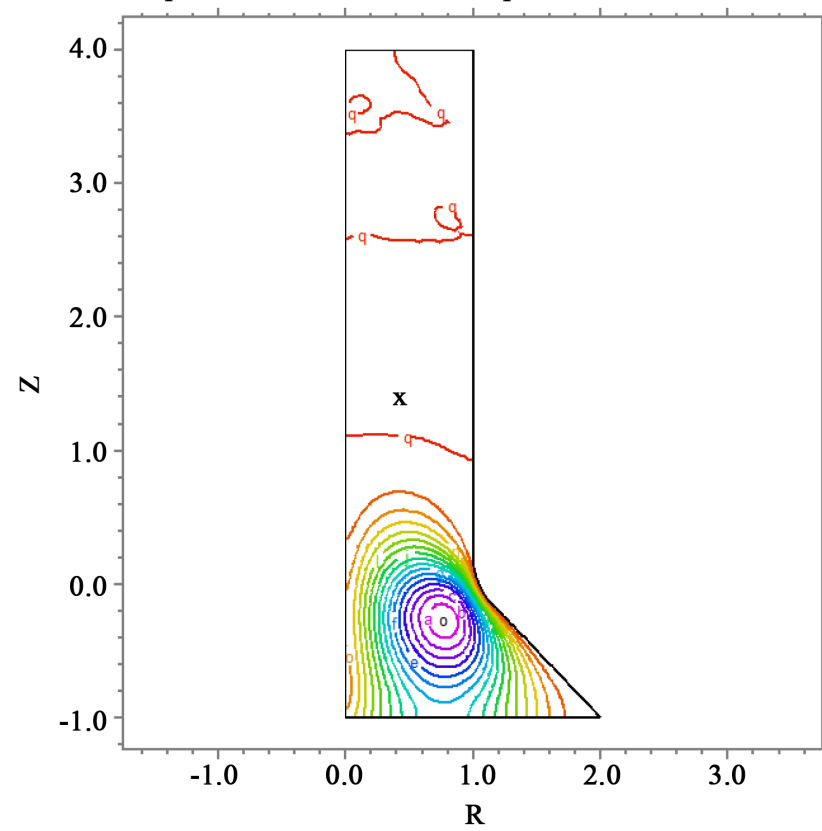

Bob4tp: Grid\#7 P3 Nodes $=5086$ Cells $=1395$ RMS Err $=7.0 \mathrm{e}-5$ Vol_Integral $=-1.281140$

Figure 4. The radial velocity contours. 
Flow Development Problem in Round Pipe

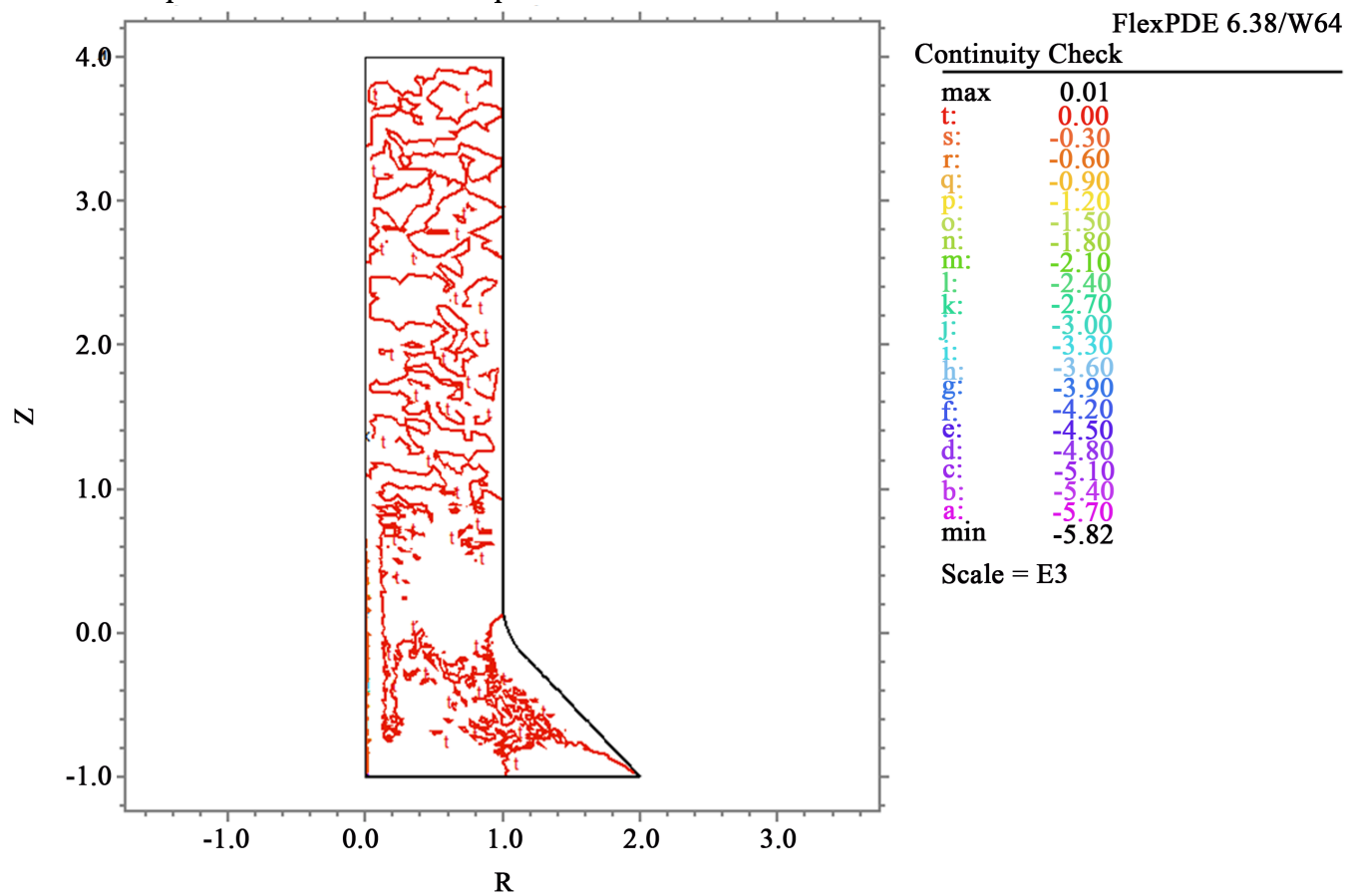

Bob4tp: Grid\#7 P3 Nodes $=5086$ Cells $=1395$ RMS Err $=7.0 \mathrm{e}-5$

Vol_Integral $=-0.061353$

Figure 5. The divergence of the velocity vector.

Flow Development Problem in Round Pipe

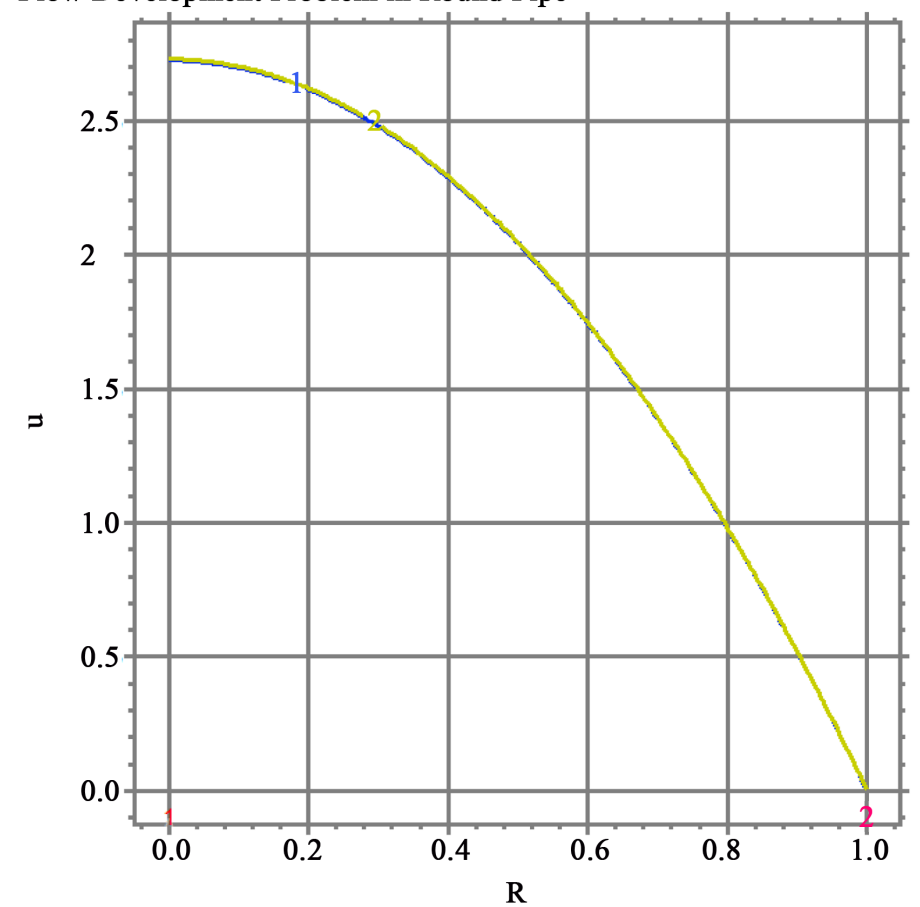

$16: 7: 464 / 8 / 16$

FlexPDE 6.38/w64 from $(0,0.62 * h)$

to $(1,0.62 * \mathrm{~h})$

1: u

2: u ex

Bob4tp: Grid\#7 P3 Nodes $=5086$ Cells $=1395$ RMS Err $=7.0 \mathrm{e}-5$ Surf_Integral $(1)=4.279831$ Surf_Integral $(2)=4.288188$

Figure 6. Comparison of fully developed and calculated flow. 
Flow Development Problem in Round Pipe

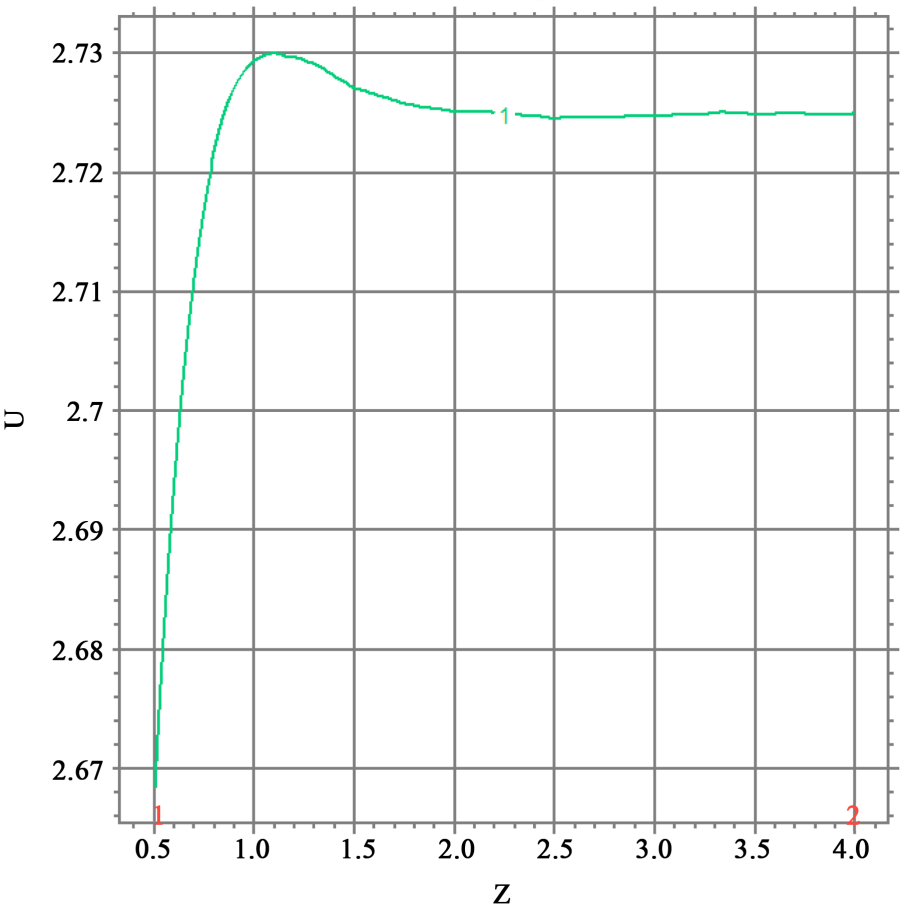

$21: 36: 526 / 4 / 16$

FlexPDE 6.38/w64
$\mathrm{U}$

from $(0,0.5)$

to $(0, \mathrm{~h})$

1: $\mathrm{U}$

Bob5tp: Grid\#7 P3 Nodes $=5086$ Cells $=1395$ RMS Err $=7.0 \mathrm{e}-5$

$0.99 *$ globalmax $(U)=2.702687$ Surf_Integral $=5.989722 \mathrm{e}-5$

Figure 7. Fluid velocity along the center of the circular pipe.

fully developed. However, the difference between the peak and the fully developed velocity is small enough in this case that the correct development length occurs close to $Z=0.62$. The size of the peak, first seen by Durst [6] et al. may depend upon the shape of the entrance to the circular pipe and every new case should be calculated without reference to a single handbook formula. This was a very difficult problem in the early days of digital computing and was the subject of several doctoral dissertations and took years of effort to solve. Now many pipe development problems can be solved with less than one day of effort.

The continuity equation is very closely satisfied in the whole region of the fluid.

\section{References}

[1] Schiller, L. (1922) Die Entwicklung der laminaren Geschwindigkeitsverteilung und ihre Bedeutung für Zähigkeitsmessungen, (Mit einem Anhang über den Druckverlust turbulenter Strömung beim Eintritt in ein Rohr.). Zeitschrift für angewandte Mathematik und Mechanik, 2, 96-106. http://dx.doi.org/10.1002/zamm.19220020203

[2] Bodoia, J.R. and Osterle, J.F. (1961) Finite Difference Analysis of Plane Poiseuille and Couette Flow Developments. Applied Scientific Research, A10, 265. http://dx.doi.org/10.1007/BF00411919

[3] Campbell, W.D. and Slattery, J.C. (1962) Flow in the Entrance of a Tube. ASME Paper No. 62-Hyd-6.

[4] Gerrad, J.H. and Taylor, L.A. (1977) Mathematical Model Representing Blood Flow Inarteries. Medical and Biological Engineering and Computing, 19, 611-617. http://dx.doi.org/10.1007/BF02457918

[5] Hornbeck, R.W. (1964) Laminar Flow in the Entrance Region of a Pipe. Applied Scientific Research, Section A, 13, 224-236. http://dx.doi.org/10.1007/BF00382049

[6] Durst, F., Ray, S., U'nsal, B. and Bayoumi, O.A. (2005) The Development Lengths of Laminar Pipe and Channel Flows. Transactions of the ASME, 127, 1154-1160. http://dx.doi.org/10.1115/1.2063088

[7] T"oppel, M., Rada, M. and Pasche, E. (2006) Hydrodynamic Flow Simulation in Pipe Networks Including Heat Transfer. Dresdener Wasserbauliche Mitteilungen, 32, 461.

[8] Hinsen, K. (2016) Scientific Communication in the Digital Age. Physics Today, 69, \#6. 
Submit or recommend next manuscript to OALib Journal and we will provide best service for you:

- Publication frequency: Monthly

- 9 subject areas of science, technology and medicine

- Fair and rigorous peer-review system

- Fast publication process

- Article promotion in various social networking sites (LinkedIn, Facebook, Twitter, etc.)

- Maximum dissemination of your research work

Submit Your Paper Online: Click Here to Submit

Contact Us: service@oalib.com 